\title{
Traveling-Wave Electrophoresis For Microfluidic Separations
}

\author{
Boyd F. Edwards, ${ }^{1}$ Aaron T. Timperman, ${ }^{2}$ R. Lloyd Carroll, ${ }^{2}$ Kyoo Jo, ${ }^{2}$ Jon M. Mease, ${ }^{1}$ and Jarrod E. Schiffbauer ${ }^{1}$ \\ ${ }^{1}$ Department of Physics, West Virginia University, Morgantown, West Virginia 26506, USA \\ ${ }^{2}$ Department of Chemistry, West Virginia University, Morgantown, West Virginia 26506, USA
}

(Received 17 July 2008; published 20 February 2009)

\begin{abstract}
Models and microfluidic experiments are presented of an electrophoretic separation technique in which charged particles whose mobilities exceed a tunable threshold are trapped between the crests of a longitudinal electric wave traveling through a stationary viscous fluid. The wave is created by applying periodic potentials to electrode arrays above and below a microchannel. Predicted average velocities agree with experiments and feature chaotic attractors for intermediate mobilities.
\end{abstract}

DOI: 10.1103/PhysRevLett.102.076103

Separations of charged substances are important in proteomics, molecular biology, cell biology, genetics, materials synthesis, and bioengineering, and are integral to microfluidic lab-on-a-chip devices that are being developed for rapid clinical and forensic analysis [1]. Over the last 25 years, capillary electrophoresis (CE) has set the standard for high-efficiency separations in solution [2]. This technique employs static, uniform electric fields to separate ions with different charge-to-size ratios into distinct zones for analysis, with zone dispersion limited ultimately by molecular diffusion.

In this Letter, we study an electrophoretic separation technique that differs from CE by trapping ions whose mobilities exceed a tunable threshold between the crests of longitudinal electric field waves traveling through a stationary solution. These waves are created by applying oscillating potentials to interdigitated arrays of stationary electrodes above and below a microfluidic channel (Fig. 1). The trapping threshold depends on the ion mobility, the electrode spacing, and the potential frequency and amplitude, and allows modulation between separative and nonseparative transport by simply varying the frequency. Separations by traveling-wave electrophoresis (TWE) (Fig. 2) apply to ions, charged biomolecules, and micronsized charged particles, and might reduce zone dispersion to below the diffusion limit.

Others use interdigitated electrode arrays on a single surface to transport charged species via electrophoresis, imposing static perpendicular gravitational or electric fields to draw particles to the surface [3-5]. Our sandwich architecture precludes such fields by bounding a microfluidic channel by electrode-bearing surfaces above and below. This design allows the use of low applied voltages to avoid unwanted electrochemical effects while keeping the electric field high to achieve rapid separations. Singlesurface architectures can also transport charged particles via ac electroosmotic pumping [6,7] and neutral bioparticles via dielectrophoresis [8].

We consider the motion of ions of charge $q$, hydrodynamic radius $r$, and velocity $\mathbf{v}$ through a stationary electri-
PACS numbers: 82.45. $-\mathrm{h}, 05.45 . \mathrm{Ac}, 82.40 . \mathrm{Bj}, 87.15 . \mathrm{Tt}$

cally conducting solution of viscosity $\eta$ and mass density $\rho$ in response to oscillating electric potentials applied to periodic arrays of electrodes. In contrast with studies of oscillator synchronization [9], TWE potentials are external functions of time. In contrast with electron trapping by plasma waves [10], viscosity is essential to TWE trapping. The 1-32 Hz frequencies of our applied potentials are too small to induce ac electroosmotic pumping, which requires frequencies of the order of $1 \mathrm{kHz}$ [6].

Models presented here include only electric and Stokes drag forces $\mathbf{F}_{E}=q \mathbf{E}$ and $\mathbf{F}_{D}=-6 \pi \eta r \mathbf{v}$ on the ions, ignoring magnetic and gravitational fields, molecular diffusion, charge redistribution, fluid flow, and ionic inertia. Setting $\mathbf{F}_{E}+\mathbf{F}_{D}=0$ immediately yields the associated electrophoretic velocity

$$
\mathbf{v}=\mu \mathbf{E}
$$

with mobility $\mu=q / 6 \pi \eta r$ and electric field $\mathbf{E}=-\boldsymbol{\nabla} \phi$.

Insight can be drawn from a $1 \mathrm{D}$ electric potential [11]

$$
\phi(x, t)=\phi_{0} \sin (k x-\omega t)
$$

with amplitude $\phi_{0}$, wave number $k=2 \pi / \lambda$, angular frequency $\omega=2 \pi / \tau$, and electric field amplitude $E_{0}=k \phi_{0}$. We define a convenient dimensionless measure of the responsiveness of an ion to the wave as

$$
R=\frac{\mu E_{0}}{c},
$$

the ratio of the characteristic electrophoretic velocity $\mu E_{0}$ to the wave speed $c=\omega / k$. Here, $q>0$ and $R>0$ for cations and $q<0$ and $R<0$ for anions.

The steady-state solution to the 1D model yields, for $|R| \geq 1$, the trapped velocity $v_{x}=c$, with trapped cations traveling within potential wells and anions within peaks. For $|R|<1$, ions experience longitudinal oscillations of angular frequency $\Omega=\omega\left(1-R^{2}\right)^{1 / 2}$ but make net forward progress with average velocity [12]

$$
\frac{\bar{v}_{x}}{c}=1-\left(1-R^{2}\right)^{1 / 2} .
$$



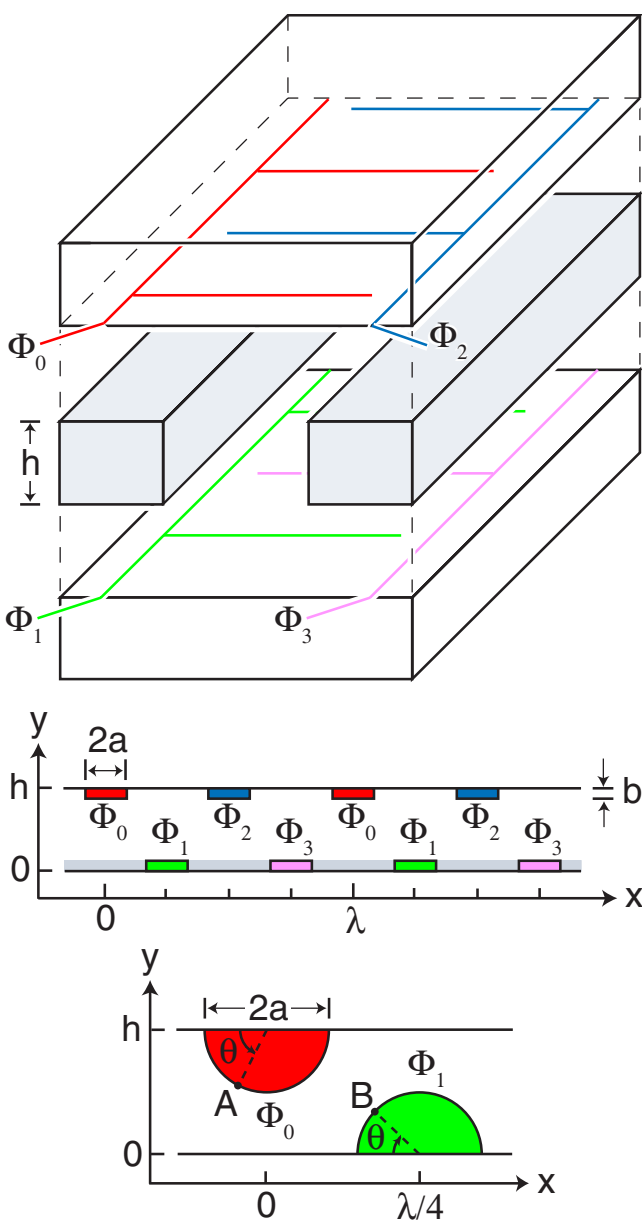

(b)

(c)

FIG. 1 (color online). (a) Exploded view of sandwich architecture for traveling-wave electrophoresis. A microchannel is formed between two glass plates (transparent) and two polydimethylsiloxane (PDMS) spacers of height $h=15 \mu \mathrm{m}$ (gray). Photolithographically patterned interdigitated arrays of gold electrodes on the bottom surface of the top plate are held at oscillating electrical potentials $\Phi_{0}(t)$ and $\Phi_{2}(t)$. Similar arrays atop the bottom plate are held at potentials $\Phi_{1}(t)$ and $\Phi_{3}(t)$. (b) Side view of the channel showing two replicates of the fourelectrode pattern, of wavelength $\lambda=80 \mu \mathrm{m}$. The experimental device has 50 replicates of this pattern and electrode cross sections of half width $a=1 \mu \mathrm{m}$ and thickness $b=150 \mathrm{~nm}$. The fabrication process leaves a PDMS layer (gray) of thickness comparable to $b$ on the bottom plate. The potential of each electrode leads its neighbor to the right in phase by $90^{\circ}$, producing a wave propagating to the right that can trap ions whose mobilities exceed a tunable threshold. (c) Detailed view of the cylindrical electrodes of radius $a=1 \mu \mathrm{m}$ used for the 2D model, whose four-electrode pattern is replicated indefinitely. Positions on electrode surfaces (points $A$ and $B$ ) are specified by contact angles $\theta$ satisfying $0 \leq \theta \leq \pi$.

During each oscillation, an ion fails to catch a passing wave and lags one cycle behind the wave, like a surfer failing to catch a passing ocean wave.

Electrodes are needed to sustain traveling waves in viscous solutions, waves that would attenuate otherwise.

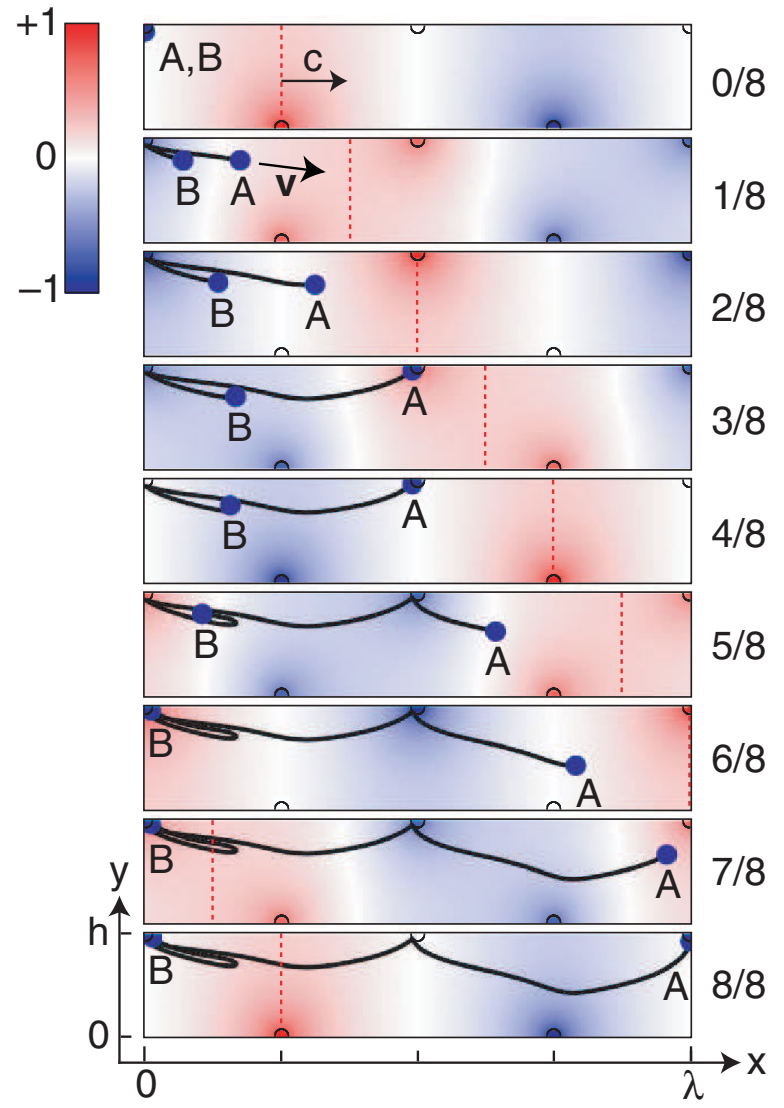

FIG. 2 (color online). Simulated separation of two ions with different mobilities and the same initial positions based on the 2D model, obtained by applying the potentials of Eq. (5) to the electrode pattern of Fig. 1(c). Shown are snapshots taken over one period $\tau$ of the wave motion, at times $t / \tau=0 / 8,1 / 8$, etc. The color bar represents values of the electric potential $\phi(x, y, t)$ relative to its amplitude $\phi_{0}$. Dashed lines identify crests of the potential that travel along the channel at average speed $c=f \lambda$, where $f=1 / \tau$ is the electrode oscillation frequency. A "trapped" high-mobility anion with responsiveness $R=-3$ $[A$, Eq. (3)] keeps pace with this crest, on average, traveling one wavelength during each period of the wave motion. A "localized" low-mobility anion with $R=-1(B)$ is unable to keep pace with the wave, oscillating instead about a single electrode. Cations with $R=3$ and $R=1$ follow the same trajectories as these anions if released at the same initial position after a phase shift of $180^{\circ}$. The ion velocity vector $\mathbf{v}$ has a time average $\overline{\mathbf{v}}=c \hat{\mathbf{x}}$ for trapped cations and anions and $\overline{\mathbf{v}}=0$ for localized ions.

Our 2D model accordingly assumes an array of long conducting cylindrical electrodes [Fig. 1(c)] held at synchronized potentials $\Phi_{0}(t), \Phi_{1}(t), \Phi_{2}(t)$, and $\Phi_{3}(t)$ given by

$$
\Phi_{i}(t)=\phi_{0} \sin \left(k x_{i}-\omega t\right)
$$

where $x_{i}=i \lambda / 4$ denotes the axial position of electrode $i=0,1,2,3$ and $\lambda=2 \pi / k$ is the electrode pattern wavelength (Fig. 1). We retain the definition of $R$ given by Eq. (3) and assume that the bulk solution is electrically 
neutral, an improvement upon the non-neutral 1D model. We solve Laplace's equation to obtain the 2D potential $\phi(x, y, t)$ within the bulk solution, with the four potentials serving as boundary conditions at the impenetrable electrode surfaces and with impenetrable channel boundaries at $y=0$ and $y=h$. This solution is a sum of logarithmic potentials for pairs of cylindrical electrodes [13] that includes image electrodes to satisfy the boundary conditions. We integrate the associated 2D nonlinear nonautonomous system of ordinary differential equations,

$$
\begin{aligned}
& \dot{x}=v_{x}(x, y, t), \\
& \dot{y}=v_{y}(x, y, t),
\end{aligned}
$$

using a fifth-order Runge-Kutta-Fehlberg method with adaptive stepsize control [14], with wavelength $\lambda=$ $80 \mu \mathrm{m}$, electrode radius $a=1 \mu \mathrm{m}$, and channel height $h=15 \mu \mathrm{m}$ pertinent to experiments. The time dependence of the velocity components $v_{x}=-\mu \partial \phi / \partial x$ and $v_{y}=-\mu \partial \phi / \partial y$ requires a $3 \mathrm{D}$ phase space for this system, the minimum dimensionality needed for chaos [15]. Previous studies of 2D nonautonomous systems [16] exclude impenetrable barriers such as our electrodes.

Relative to the 1D model (Fig. 3, dashed trace), the average axial steady-state velocity $\bar{v}_{x}$ for the 2D model (Fig. 3, solid trace) exhibits (i) a larger trapping threshold $R_{t}=2.58$ reflecting incomplete penetration of the potential into the channel and (ii) a nonzero localization threshold $R_{l}=1.10$ below which low-mobility ions oscillate about electrodes $\left(\bar{v}_{x}=0\right)$. The 2D wave traps ions whose

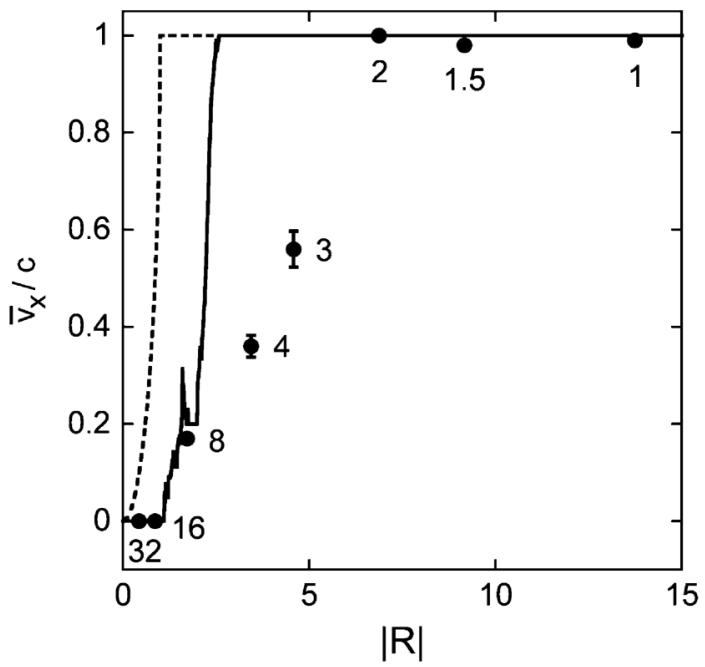

FIG. 3. Ratio of the steady-state average axial ion velocity $\bar{v}_{x}$ to the wave speed $c$ vs the responsiveness $R$, from Eq. (4) for the $1 \mathrm{D}$ model (dashed trace), for the 2D model (solid trace), and for microfluidic experiments (data points accompanied by numerical values of the frequency $f$ ). In the experiments, ions are trapped for $f=1,1.5$, and $2 \mathrm{~Hz}$, partially trapped for $f=3,4$, and $8 \mathrm{~Hz}$, and localized for $f=16$ and $32 \mathrm{~Hz}$. The 2D model captures the localization threshold and underestimates the trapping threshold. mobilities $|\mu|$ exceed the tunable threshold mobility $\mu_{t}$ defined by $R_{t}=\mu_{t} E_{0} / c$.

These $2 \mathrm{D}$ predictions agree with measurements of the velocities of fluorescein plugs traveling along the TWE channel of Figs. 1(a) and 1(b) with fixed $\phi_{0}=0.5 \mathrm{~V}$ and variable frequency $f=1 / \tau$ (Fig. 3, data points). Plugs containing $20 \mu \mathrm{M}$ fluorescein and $10 \mu \mathrm{M}$ sodium phosphate were injected electrokinetically into the channel, which was filled with a $10 \mu \mathrm{M}$ sodium phosphate running buffer. A six-step stepwise-constant periodic potential that mimics Eq. (5) was then applied to the electrodes. Average plug velocities $\bar{v}_{x}$ were determined microscopically by measuring the time of passage of the peak fluorescein intensity between two fixed locations separated by at least $400 \mu \mathrm{m}$. Values of $c$ follow from $c=f \lambda$ and values of $R$ from Eq. (3) using the fluorescein mobility $\mu=-2.8 \times$ $10^{-4} \mathrm{~cm}^{2} / \mathrm{V} \mathrm{s}$ determined from CE experiments. Each data point in Fig. 3 is centered at the average of two independent measurements of $\bar{v}_{x} / c$, with the ends of the error bars showing these two measurements when they differ by more than the diameter of the plotting symbol.

TWE might reduce the dispersion of trapped analyte plugs to below the diffusion limit. At $f=1 \mathrm{~Hz}$, a fluorescein plug takes $t=50 \mathrm{~s}$ to travel the length of the $4 \mathrm{~mm}$ TWE channel. During this time, molecular diffusion would increase the full width at half maximum of the plug by [2] $\Delta \sigma=(2 D t)^{1 / 2}=0.2 \mathrm{~mm}$, where $D=k_{B} T \mu / q=$ $3.56 \times 10^{-6} \mathrm{~cm}^{2} / \mathrm{s}$ is the fluorescein diffusivity, $q=$ $-3.2 \times 10^{-19} \mathrm{C}$ is its charge, $k_{B}$ is Boltzmann's constant, and $T=295 \mathrm{~K}$ is the temperature. Preliminary measurements show that fluorescein plugs of initial width $1.3 \mathrm{~mm}$ spread less than $0.2 \mathrm{~mm}$, indicating that TWE might elute bands with little or no dispersion.

Partially trapped ions exhibit (i) periodic and narrow band chaotic attractors with commensurate velocities $\bar{v}_{x} / c=1 / 5$ and $1 / 9$, (ii) broadband chaotic attractors with incommensurate velocities, and (iii) period-doubling cascades to chaos (Fig. 4). Illustrated in the inset of Fig. 4 are a period-1 attractor, a narrow band chaotic attractor, and a broadband chaotic attractor. Ions on the period-1 attractor follow identical paths between adjacent electrodes, apart from simple translations and reflections, each path including one lag cycle and contacting an electrode at a specific angle, $\theta=0.65 \pi$. Ions on the narrow band chaotic attractor follow similar paths and contact electrodes somewhere in the range $0.49<\theta / \pi<0.88$. Ions on the broadband chaotic attractor travel between electrodes in a variety of ways and sample the entire range of angles, $0<\theta<\pi$. Such broadband chaotic attractors exhibit self-similar nested sequences that converge at unstable trapped orbits [17].

Future explorations might include experiments with charged tracer particles and models that include ion diffusion, fluid flow, and charge redistribution. Neglecting diffusion is justified by the large Péclet numbers $P=\lambda c / 2 D$ 

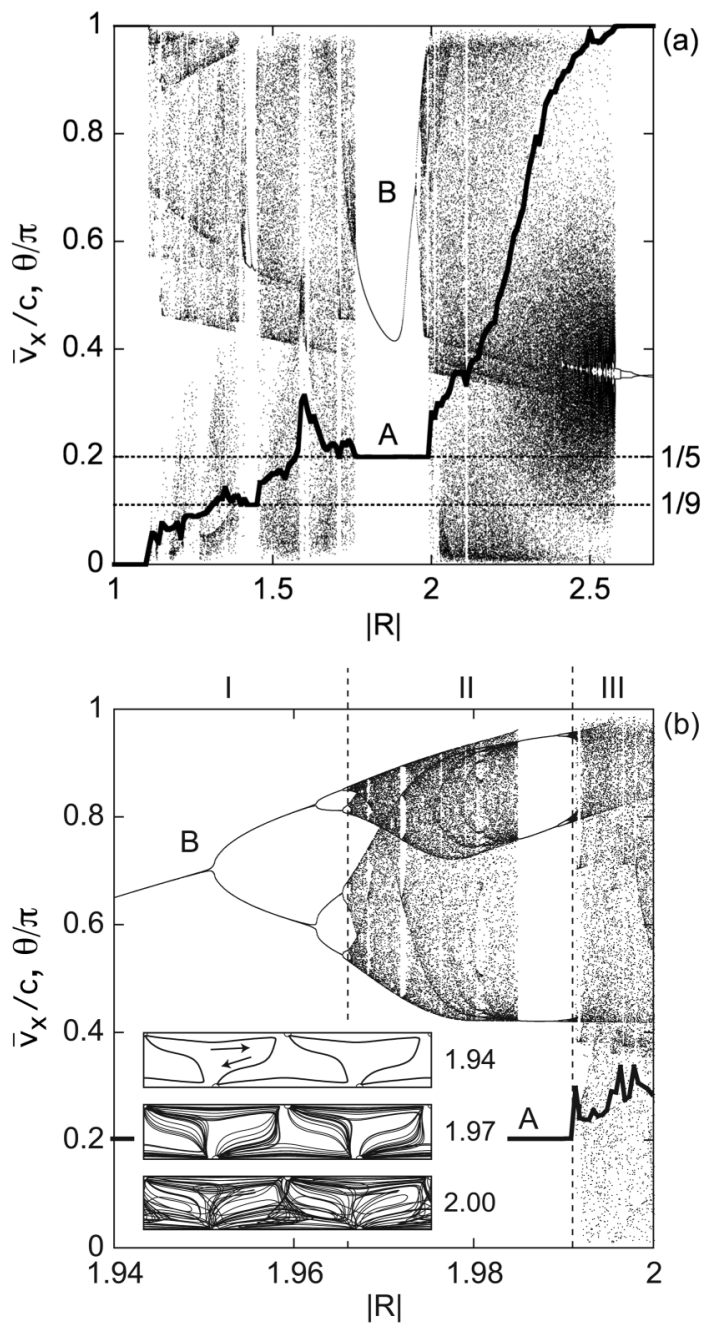

FIG. 4. (a) Details of $\bar{v}_{x} / c$ vs $R$ from the 2D model (heavy trace, $A$ ) and steady-state values of the electrode contact angle $\theta$ for simulations at fixed $R(B)$. Chaotic attractors feature scattered values of $\theta$ whose specific positions vary from simulation to simulation. Periodic attractors feature one or more isolated values. (b) Detail near $|R|=2$ showing a period-doubling cascade with $\bar{v}_{x} / c=1 / 5$ (I), narrow band chaos with periodic windows with $\bar{v}_{x} / c=1 / 5$ (II), and broadband chaos with incommensurate velocities (III). Inset: Steady-state trajectories wrapped to the interval $0 \leq x \leq \lambda$, including a period- 1 attractor for $|R|=1.94$, a narrow band chaotic attractor for $|R|=1.97$, and a broadband chaotic attractor with $\bar{v}_{x} / c=0.283$ for $|R|=$ 2.00.

satisfying $10<P<300$, though diffusion might refine predictions of the trapping threshold. Our low frequencies $f$ preclude net electroosmotic pumping of fluid along the microchannel axis [6], but might allow localized circulatory electroosmotic flow (EOF) because $\tau=1 / f \approx 0.1 \mathrm{~s}$ is large compared with the characteristic EOF response time $\tau_{\mathrm{EOF}}=4 h^{2} \rho / \eta \approx 0.0003 \mathrm{~s}$ [18]. We intend to study the extent and effects of such flows via models and experiments with uncharged tracer particles.

We gratefully acknowledge support from NSF Grants No. DMR-0647763 and No. EPS-0554328, a WVU Research Corporation PSCoR grant, technical assistance from John Edwards and Kathleen Kelly, and helpful conversations with Mark Koepke, David Lederman, Earl Scime, Kenneth Showalter, James Vopal, and Arthur Weldon.

[1] E. Verpoorte, Electrophoresis 23, 677 (2002).

[2] J.W. Jorgenson and K. D. Lukacs, Science 222, 266 (1983).

[3] S. Masuda, M. Washizu, and M. Iwadare, IEEE Trans. Ind. Appl. IA-23, 474 (1987).

[4] H. Kawamoto and S. Hayashi, J. Phys. D 39, 418 (2006); H. Kawamoto, K. Seki, and N. Kuromiya, J. Phys. D 39, 1249 (2006), and references therein.

[5] M. H. Lean, H. B. Hsieh, and A. R. Volkel, in Proceedings of the 3rd Annual International IEEE EMBS Special Topic Conference on Microtechnologies in Medicine and Biology, 2005 (IEEE, New York, 2005), p. 356; M. H. Lean, A. R. Volkel, H. B. Hsieh, J.-P. Lu, J. H. Daniel, B. T. Preas, and S. J. Limb, ibid., p. 80.

[6] A. Ramos, H. Morgan, N. G. Green, A. Gonzalez, and A. Castellanos, J. Appl. Phys. 97, 084906 (2005).

[7] R. M. Ehrlich and J. R. Melcher, Phys. Fluids 25, 1785 (1982).

[8] H. Du, W. H. Li, D. F. Chen, and C. Shu, Int. J. Mech. Mat. Des. 1, 115 (2004).

[9] A. Pikovsky, M. Rosenblum, and J. Kurths, Synchronization: A Universal Concept in Nonlinear Sciences (Cambridge, New York, 2003).

[10] R. N. Franklin, S. M. Hamberger, H. Ikezi, G. Lampis, and G. J. Smith, Phys. Rev. Lett. 28, 1114 (1972).

[11] H.-H. Wei, Appl. Phys. Lett. 90, 204103 (2007).

[12] J. R. Melcher, E. P. Warren, and R. H. Kotwal, Part. Sci. Technol. 7, 1 (1989).

[13] R. K. Wangsness, Electromagnetic Fields (Wiley, New York, 1986), Sec. 5-3.

[14] W. H. Press, S.A. Teukolsky, W. T. Vetterling, and B. P. Flannery, Numerical Recipes in $\mathrm{C}++$ (Cambridge University Press, New York, 1992), function ODEINT, Sec. 16.2.

[15] S. H. Strogatz, Nonlinear Dynamics and Chaos (AddisonWesley, New York, 1994), Sec. 1.2.

[16] N. A. Magnitskii and S. V. Sidorov, Differ. Equ. 42, 1579 (2006).

[17] B. F. Edwards (unpublished).

[18] D. Yan, N.-T. Nguyen, C. Yang, and X. Huang, J. Chem. Phys. 124, 021103 (2006). 